\title{
Caracterización del emprendimiento desde un enfoque universitario
}

\author{
Carlos Pacheco-Ruiz', Claudia Rojas-Martínez', William Niebles-Nuñez', Hugo G. Hernández-Palma* \\ (1) Universidad de Sucre, Programa de Administración de Empresas, Sincelejo-Colombia. \\ (correo-e: carlos.pacheco@unisucre.edu.co; claudia.rojas@unisucre.edu.co; william.niebles@unisucre.edu.co) \\ (2) Universidad del Atlántico, Programa Administración de Empresas, Barranquilla-Colombia. \\ (correo-e: hugohernandezpalma@gmail.com) \\ ${ }^{*}$ Autor a quien debe ser enviada la correspondencia.
}

Recibido Jun. 30, 2021; Aceptado Ago. 26, 2021; Versión final Oct. 7, 2021, Publicado Feb. 2022

\section{Resumen}

Esta investigación buscó analizar las acepciones vinculadas al emprendimiento, articulando esta indagación con las necesidades y expectativas que actualmente se vienen exponiendo al respecto. La metodología fue de corte descriptivo y se realizó un análisis detallado de la literatura. Se seleccionaron 20 fuentes de información publicadas en Scopus, Scielo o Web of Science. Los resultados exponen que en la medida que desde la universidad se desarrollen procesos tempranos, existen mayores posibilidades para la generación del emprendimiento por parte del estudiante o futuro profesional. Se identifican también los perfiles y modelos de emprendimiento que han marcado hitos en el proceso histórico. Se concluye que la responsabilidad de los proyectos de emprendimiento, que lleven a su éxito o fracaso, no debe depender exclusivamente del emprendedor, puesto que los contextos sociales y económicos de la actualidad propician una bilateralidad con respecto al apoyo que debería presentarse por parte de otros actores involucrados.

Palabras clave: emprendimiento; educación superior; innovación; creatividad; universidad

\section{Characterization of entrepreneurship from a university approach}

\begin{abstract}
The main objective of this study was to assess the different definitions of "entrepreneurship" by conducting an inquiry according to current needs and expectations. The methodology was descriptive, and a detailed literature review analysis is performed. Twenty published sources were selected from Scopus, Scielo, and Web of Science. The results showed that the sooner entrepreneurship processes develop at universities, the greater the opportunities that students and future professionals have for generating successful entrepreneurships. The results also revealed entrepreneurial profiles and models of historical importance. It is concluded that what leads entrepreneurial projects to success or failure should not depend exclusively on entrepreneurs since the social and economic contexts of today create a bilateral responsibility with respect to the support that entrepreneurs should receive from other factors involved.
\end{abstract}

Keywords: entrepreneurship; higher education; innovation; creativity; university 


\section{INTRODUCCIÓN}

Cuando se habla del concepto de emprendimiento, se puede hacer un acercamiento al término desde una perspectiva etimológica, la cual, según lo expuesto por Bernal et al., (2015), permite la comprensión de algunas de las características más destacadas respecto a su significado, alcance, evolución y propósito, que han sido estudiadas por numerosos autores en los últimos años, en un esfuerzo por comprender de manera holística este fenómeno que hoy día cuenta con un papel importante en muchos sectores de la sociedad, especialmente en el educativo. En este sentido, se denota lo estipulado por Herrera y Montoya (2013), quienes expresan que el término emprendimiento (entrepreneurship) tiene sus raíces en la palabra "entreprendre" de origen francés y el vocablo alemán "unternehmen", la unión de estas dos palabras dio paso al término que hoy día se conoce como emprendedor. Inicialmente, los franceses fueron quienes dieron a la palabra una connotación que estaba relacionada con los pioneros, exploradores y personas que se aventuraban en la búsqueda de lo desconocido.

En este orden de ideas, posteriormente, según las investigaciones de Brown y Thorton (2013), a mediados del año 1700, el economista irlandés Richard Cantillon fue el primero que logró dar un cuerpo desde el carácter conceptual a lo que significaba el emprendimiento y, en tal sentido, lo describió como la capacidad que tienen los individuos para desarrollar nuevas formas de hacer y pensar para materializar un objetivo, asumiendo los riesgos que esto conlleva. Seguidamente, se destaca lo expresado por Numa (2019) quien expone el aporte del economista y empresario francés Jean Baptiste Say fue de especial importancia, pues expuso una característica diferenciadora entre el capitalista y el emprendedor, dado que éste es quien cuenta con la capacidad para reunir y administrar medios de producción y convertirlo en un proyecto productivo sostenible, mientras que el capitalista solo jugaba un papel de aportante de recursos, que distaba con la creación y motivación para finalizar un procesos una vez iniciado.

De esta manera, y según la línea de tiempo de evaluación del concepto, se encuentra que a principios del siglo XX, las ideas de Schumpeter en 1935 sobre el emprendedor fueron más completas, en el sentido que dio al emprendedor características únicas y extraordinarias que le permitían generar innovación capaz de reformar los patrones de producción y reorganizar la industria (Bittencourt, 2012); bajo esta concepción, el emprendedor se puede entender como una persona extraordinaria con alta capacidad para crear, renovar, afianzar y sorprender con ideas y soluciones ante situaciones de mercados de características diversas (Becker, 2012).

La mencionada concepción del emprendedor como generador de innovación en el mercado es sostenida por Raposo y do Paco (2011), autores quienes exponen que la innovación es un componente esencial de los emprendedores, puesto que con procesos innovadores, estos no solo son capaces de aprovechar las oportunidades para generar cambios, sino que estos cambios entran de forma contundente al entorno y crean nuevas formas de producción, dando a sus iniciativas un perfil de mayor impacto económico y social. Siguiendo con la anterior argumentación, con respecto a esta evolución, otro aporte importante en la construcción del concepto de emprendimiento es la de Bethencourt Vries (1977), citado por Bucardo et al., (2015), quien señala que, este cumple tres roles básicos al momento de generar y ejecutar la idea de emprendimiento: el rol innovador, la administración del proceso y la toma de riesgos; puesto que el emprendedor no solo arriesga recursos económicos sino también arriesga su imagen y factores psicológicos asociados al proceso de emprendimiento (Bucardo et al., 2015).

Con base en lo anterior, Dees (1998), citado por Carraher et al., (2016), complementa muy bien las características que definen el emprendimiento a partir de las ideas de otros autores y las describe como un cataclismo creativo que genera creación de valor, la identificación de oportunidades y el ingenio. Así las cosas, el autor toma cada una de estas características o ideas centrales propuestas por los investigadores mencionados y las interrelaciona y desarrolla más profundamente, a fin de obtener una visión más completa de lo que significa el emprendimiento (Carraher et al., 2016). Más recientemente, el Global Entrepreneurship Monitor (GEM), uno de los estudios de mayor relevancia y aceptación a nivel mundial con respecto a temas de emprendimiento e investigación de aspectos individuales del emprendedor y su contexto, define el emprendimiento como un proceso, en donde intervienen diversidad de elementos tales como la innovación, motivación, aspiraciones, competitividad, entre otras, que es llevado a cabo por un individuo o un grupo de estos llamados emprendedores (Tunali y Sener, 2019).

Consecuentemente se desarrolla la presente investigación con el objeto de realizar un análisis de aquellos conceptos vinculados al emprendimiento como factor primordial, pero del mismo modo, articulando esta indagación con las necesidades y expectativas que actualmente se vienen exponiendo sobre el tema desde el ámbito de la educación superior en su papel como impulsor de la proyección emprendedora entre los estudiantes universitarios. 


\section{METODOLOGÍA}

La investigación desarrollada se categoriza como tipo descriptiva, en virtud de que se hace una descripción detallada de la literatura que comprende el emprendimiento y sus características principales; así mismo, se puede esbozar un detallado, reflexivo y profundo desarrollo en el tema en cuestión de como la innovación en los diversos procesos de las instituciones de educación superior puede llegar a tener una alta influencia e impulso para los proyectos del emprendimiento del estudiante universitario (Niebles et al., 2016). También se considera un estudio documental, dado que se ha realizado una revisión y análisis de documentos escritos relacionados con el tema objeto de estudio. En este sentido autores como Hurtado de (2010) citado por Pitre et al., (2017), han enunciado que al examinar este tipo de fenómenos son pertinentes las investigaciones que se basan en la revisión documental, pues la misma permite analizar la situación o problema de origen y a partir de este proceso generar conocimientos o aportes para consultas posteriores.

En otro orden de ideas, el estudio se caracterizó por ser una investigación no experimental, dado que la variable: emprendimiento, no fue manipulada por los investigadores, tomando sus cualidades y características tal y como se perciben en el contexto de los documentos y publicaciones revisadas. De acuerdo con el estudio que se ejecutó, se realizó el proceso de recolección de datos, bajo la revisión bibliográfica, cuyo objetivo fue hacer revisión exhaustiva de los documentos que sirven de base para obtener los resultados. Asimismo, se utilizó la técnica del fichaje, considerando que esta es una herramienta que en los procesos de investigación se utiliza en función de respaldar toda la información que hayan revisado, haciendo en este contexto una caracterización bibliográfica.

De acuerdo con lo planteado anteriormente, se utilizaron fuentes de información científica tomando en consideración, tanto las publicaciones derivadas de procesos similares en organizaciones económicas, como también aquellas que proceden de universidades, el gobierno y articuladas con los procesos de emprendimiento de hoy día de los últimos diez años, tanto a nivel nacional como internacional. La recolección de la información se realizó en un solo momento y el propósito esencial fue describir la variable previamente definida; de esta manera se seleccionaron de manera intencional, 20 fuentes de información las cuales fueron ubicadas a través de la plataforma Google Scholar, y que cumplieran con las características anteriormente expresadas, pero además con las siguientes: que estuviesen publicadas en revistas de asociadas a las bases de Scopus, Scielo o Web of Science, presentadas en inglés o español, y que atendieran a los siguientes descriptores de búsqueda: Emprendimiento, Tipos de emprendimiento, Modelos formativos y Emprendimiento universitario.

De esta manera, se esboza que luego de recolectar la información se desarrolló el procedimiento de caracterización de la propia información en función de la variable emprendimiento, para lo cual se utilizó el método cualitativo, para establecer las cualidades del emprendimiento según lo que han expuesto los diversos autores seleccionados, para realizar un desglose que vincule el emprendimiento como herramienta a impulsar a través de las instituciones de educación superior. Finalmente, se tiene que con la caracterización desarrollada se da apertura a los resultados de la investigación, la cual se presenta detalladamente en el siguiente apartado.

\section{RESULTADOS Y DISCUSIÓN}

Al abordar los resultados de la revisión de literatura, se parte del planteamiento que estipulan Denicolai et al., (2015), quienes indagan sobre si las características y competencias de los individuos que deciden emprender se pueden adquirir mediante procesos de formación, o si estos factores son propios de cada persona. El perfil emprendedor cuenta con una serie de rasgos que permiten que elementos como la motivación o la capacidad de percepción de oportunidades del entorno, sean de vital importancia a la hora de desarrollar una idea (Rodríguez y Borges, 2018).

Estas características han sido revisadas y estudiadas por numerosos autores, motivados por conocer cuáles de estos factores son determinantes en un individuo para llevar a cabo ideas una idea de negocio con éxito, (Wales, 2016). La motivación para desarrollar una idea de negocio es una característica esencial de todo emprendedor, la cual, según diversos estudios, se basa en los aspectos de deseo de independencia, obtener mayores recursos económicos, satisfacción personal, necesidad de logro, entre otros (Suárez y Pedrosa, 2016). Al momento de tomar la decisión sobre iniciar un nuevo negocio, los factores relacionados con el logro personal tienen en algunos casos mayor influencia que los factores asociados con la generación de recursos económicos (Omerzel y Kušce, 2013). Al respecto, los trabajos desarrollados por McClelland (1961), citado por Obschonka et al., (2011), específicamente sobre su libro la Teoría de las Necesidades 1961 argumentan que las personas toman sus decisiones con base en los niveles de motivación que se tengan, esta motivación puede ser de tres tipos y se ilustran seguidamente en la figura 1. 


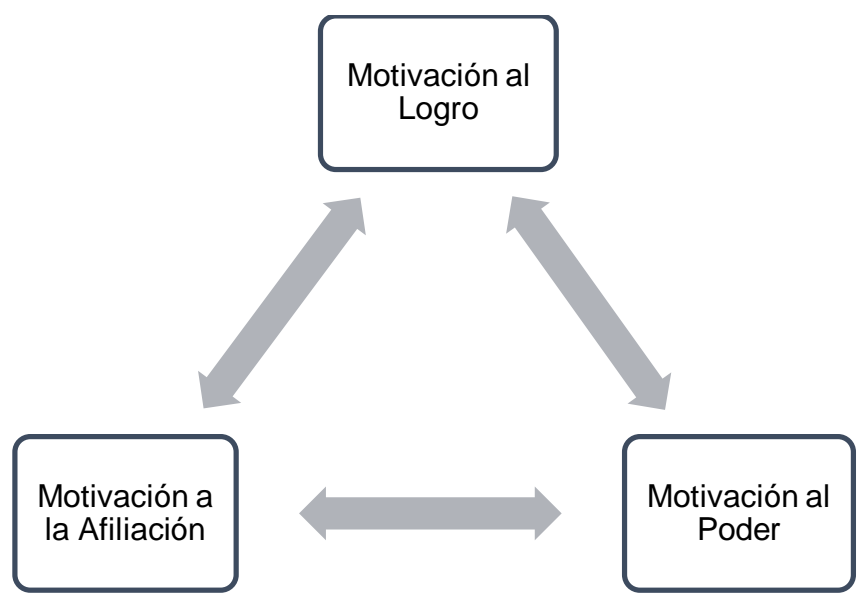

Fig 1. Tipología de motivación en emprendimiento. (Adaptada de Obschonka et al., 2011)

Como se aprecia en la figura 1, McClelland encontró que las personas que contaban con altos niveles de motivación al logro también estaban vinculadas a actividades empresariales y, por tanto, llegó a la conclusión de que si se desea crear personas que impulsen proyectos productivos lo que se debe hacer es entender cuál es su proceso de motivación, así también describió como el querer pertenecer a un grupo selecto y ostentar una posición diferencial o de poder, podría convertirse en un motor para promover iniciativas en este aspecto (Obschonka et al., 2011). Este hallazgo resulta relevante dado que el autor, a través del estudio de los períodos de desarrollo de algunos países, determinándose que existía una relación entre el desarrollo logrado por estos países y la educación impartida y, con base en esto, se formuló la hipótesis sobre la cual, si a un individuo se le forma desde temprana edad para que desarrolle una alta motivación al logro, probablemente éste se convierta en un empresario exitoso (Zhang et al., 2014).

Esta hipótesis fue comprobada en diversas culturas y países, en consecuencia, llama a la reflexión el hecho de que, si se desea lograr un desarrollo importante a nivel económico y social, se deben hacer mayores esfuerzos por crear mecanismos para fomentar un alto nivel de logro en los estudiantes y esto solo se logra a través de la educación (Singh et al, 2011). Por tal razón, si la misión de las instituciones de educación superior es la de formar profesionales integrales con altas capacidades que les permitan afrontar el mundo laboral, a través del mejoramiento de sus competencias y habilidades entorno al emprendimiento debe ser un elemento expreso en los diseños curriculares, además, implicaría un cambio de paradigma en cuanto a fomentar la cultura emprendedora dentro de la institución (Campuzano et al., 2017).

Consiguientemente con lo anterior, se tiene una diversidad de enfoques acerca de las competencias y habilidades que debe tener un emprendedor, sin embargo, algunas de las más importantes son: la autoconfianza, la orientación al logro, poseer baja propensión al riesgo, las expectativas de control, la tolerancia a la frustración y la orientación, cada una de estas características conforman la caja de herramientas necesarias a nivel personal para poder impulsar proyectos productivos, y es allí en donde particularmente el ámbito educativo puede, con base en políticas institucionales, crear un espacio para su desarrollo y fomento (Salinas y Osorio, 2012).

De forma más amplia, la Corporación Andina de Fomento (CAF) citado por Grisales (2013), propone un conjunto de características y competencias de todo emprendedor que están asociadas al logro, compromiso y liderazgo. Adicionalmente, se debe contar con la capacidad para transformar ideas en negocios o proyectos viables, puesto que la sola idea de negocios no es suficiente para considerarse emprendedor, se requiere de un conjunto de herramientas que permita pasar de la idea a la puesta en marcha y sobreponerse en cada una de las etapas donde los resultados pueden ser diferentes a los que inicialmente se proyectaron (Grisales, 2013).

Siguiendo con lo expuesto por la Corporación Andina de Fomento, dentro de las competencias asociadas al logro, se encuentra la búsqueda de oportunidades, perseverancia, calidad, toma de riesgos y tolerancia a la incertidumbre, estos rasgos permitirán, mantener una alta motivación para alcanzar los objetivos que se plantee el emprendedor (Grisales, 2013). En este orden de ideas, entre las competencias vinculadas con los compromisos, se tiene la fijación de objetivos, búsqueda de información y la elaboración de planes con seguimiento y control, en este aspecto cabe destacar que el emprendedor, no solo debe tener motivación, sino disciplina para gestionar su emprendimiento con base en objetivos, metas, planificación y mecanismos de seguimiento y control bien establecidos. Por último, las competencias del liderazgo están asociadas a la construcción de redes, la capacidad de persuasión, el trabajo en equipo, solución de problemas, negociación, iniciativa, independencia de criterio y la autoconfianza. 


\section{Tipos de emprendimiento}

A partir de lo planteado por las investigaciones consultadas, se puede decir que el emprendimiento es un proceso impulsado y materializado por los emprendedores, cuyas características, habilidades y competencias pueden variar de acuerdo con el contexto en el que se desenvuelve el individuo, a su formación o el interés y motivación por el cual decide emprender una idea de negocios.

Por lo tanto, se establece de esta manera que los emprendedores pueden ser clasificados en grupos o categorías, con respecto a ciertas características comunes que los individuos presentan en sus emprendimientos; de este modo, se presenta a continuación, en la figura 2 la clasificación de los diversos tipos de emprendimientos. De acuerdo con el esquema citado, se muestra que en primera instancia están aquellos emprendedores que desarrollan un producto o servicio innovador y que tienen sobre el mismo la propiedad intelectual del activo; seguidamente se encuentran dos grupos importantes: aquellos emprendedores que deciden poner en marcha una idea de negocios por necesidad y lo que lo hacen por oportunidad (Cantón et al., 2014).

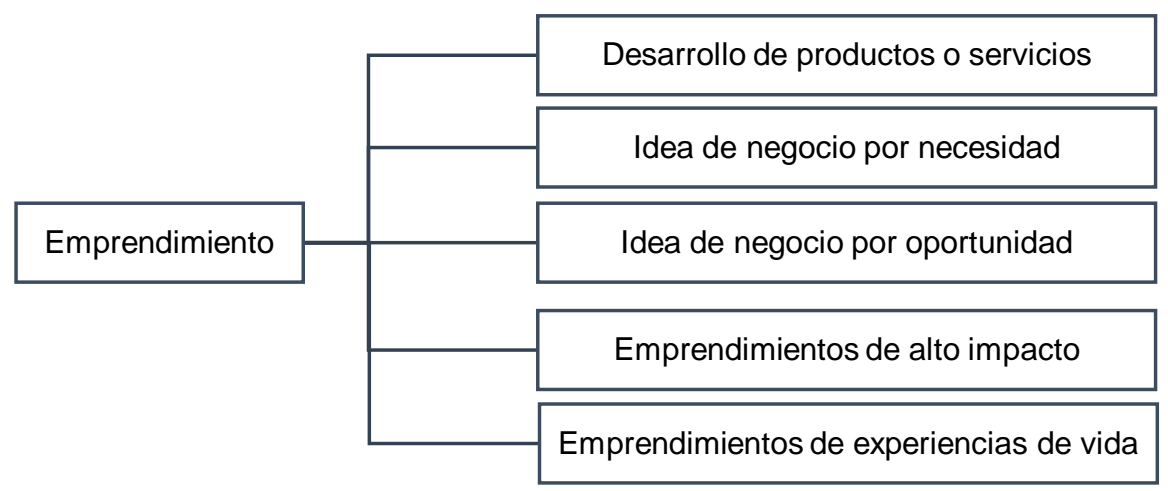

Fig 2. Tipos de emprendimiento. (Adaptada de Cantón et al., 2014)

Seguidamente, un cuarto grupo está compuesto por aquellos individuos que generan emprendimientos de alto impacto que producen innovaciones llamativas en el mercado y en consecuencia obtienen un crecimiento acelerado en sus primeros años, ejemplo de estos son Jobs y Gates en el sector de los dispositivos tecnológicos y el último grupo de emprendedores que desarrollan sus ideas basados en su proyecto de vida, los cuales se inician mayormente para poner en práctica los conocimientos y estilos de vida del emprendedor (Cantón et al., 2014).

Todos estos tipos de emprendedores suponen unas características únicas que pueden ser desarrolladas con el fin de que los proyectos productivos que éstos impulsen se conviertan en un motor para el crecimiento económico y social. Los retos de la sociedad globalizada imponen nuevas formas de pensar y actuar acordes con el contexto en el que se desarrollan, avanzando así hacia el crecimiento sostenible que la sociedad actual demanda. El sector educativo, como protagonista del desarrollo integral de futuros profesionales tiene la responsabilidad de formular y articular políticas, estrategias, acciones que fomenten espacios para el crecimiento productivo y social (Niebles et al., 2016). En este orden de ideas, el GEM, ha evaluado el perfil educativo de los empresarios establecidos contrastando con los emprendedores, mostrando los datos expuestos en la figura 3 (Tunali y Sener, 2019).

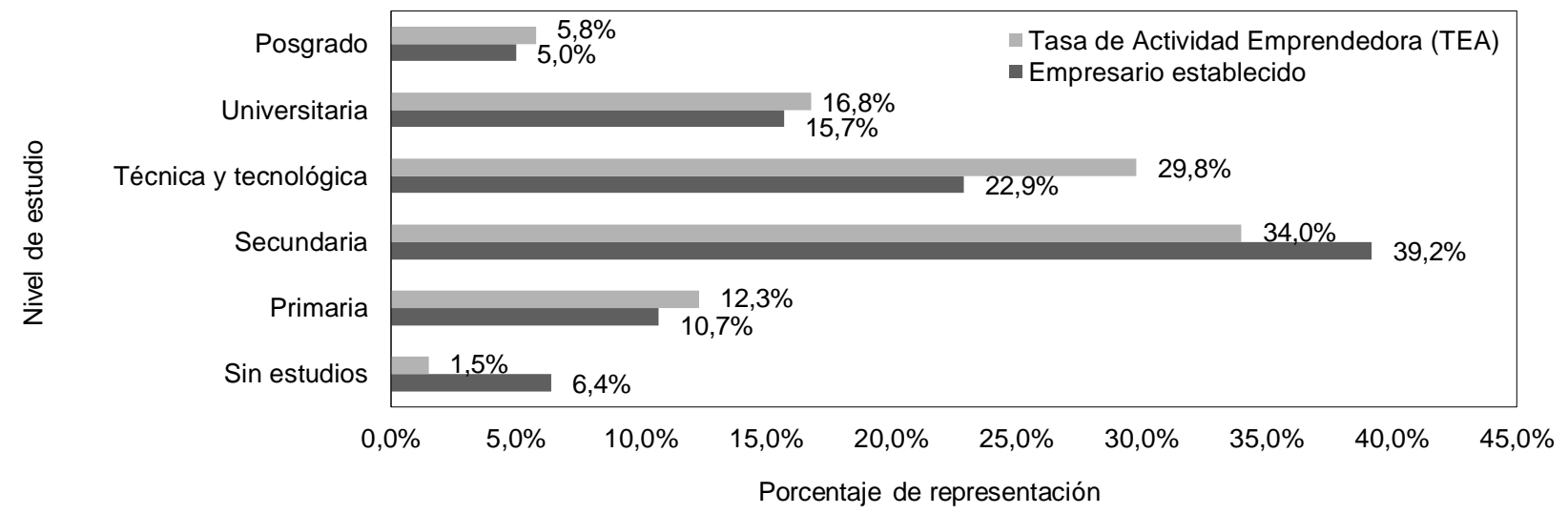

Fig 3. Nivel educativo predominante en empresarios establecidos y emprendedores a nivel mundial según GEM en 2017. (Datos de Tunali y Sener 2019) 
En virtud de lo expuesto anteriormente, se observa que el grupo de estudiantes universitarios (cursantes de pregrado y cursantes de posgrado) que son empresarios establecidos o inician un nuevo negocio medido a través de la TEA, se presentan como minoritarios con respecto a otros estudiantes como los de carreras técnicas y aquellos que han terminado el grado de secundaria, lo que indica que se presenta una oportunidad para desarrollar procesos de fortalecimiento del emprendimiento estudiantil a partir de las instituciones de educación superior, de manera que esta proporción porcentual se incremente.

En este orden de ideas, la universidad está llamada a formar a estos segmentos para fomentar el desarrollo de proyectos productivos desde la academia, con base en la implementación de estrategias de enseñanza y programas que fortalezcan las competencias y habilidades emprendedoras en los estudiantes, así como también, el apoyo en el proceso emprendedor, con miras a potenciar una educación empresarial a nivel superior (Peñaherrera y Cobos, 2012). Consiguientemente, en cuanto a las áreas prioritarias a donde se debe dirigir la atención, se pueden citar las cifras aportadas por el Fondo Emprender citadas en el trabajo de Cajigas et al., (2019), las cuales son expuestas en la figura 4, donde se aprecian el desglose porcentual de las empresas apoyadas por dicha entidad en Colombia, según su rubro especifico entre los años 2003 y 2018.

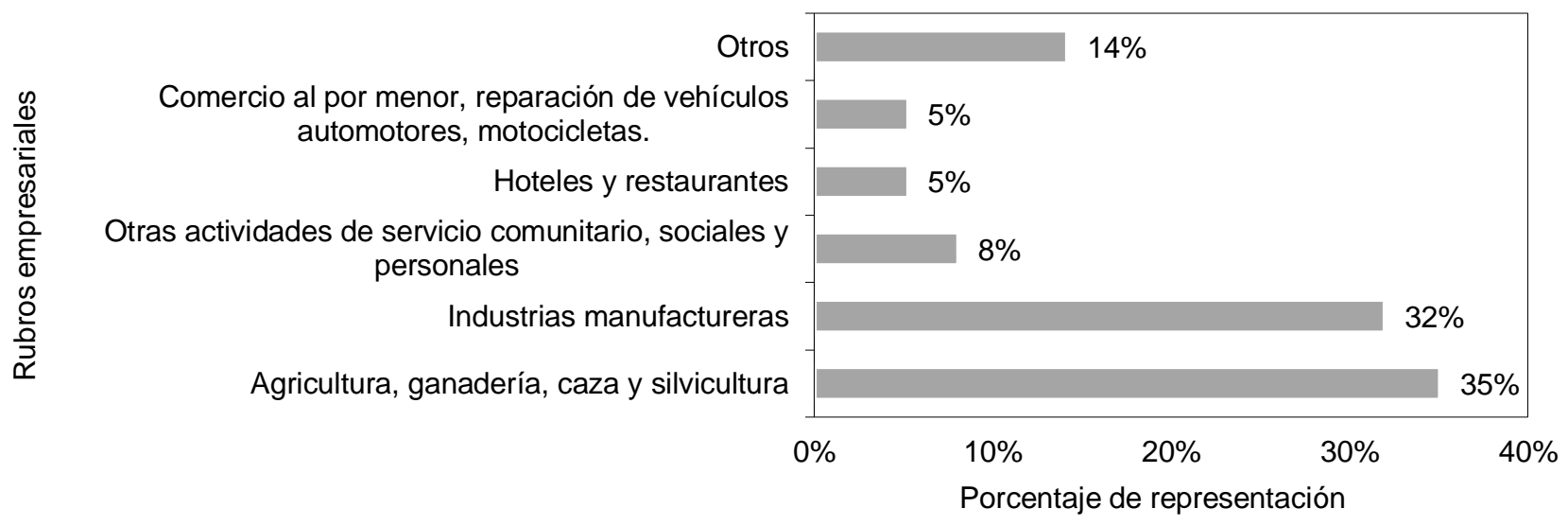

Fig. 4. Empresas apoyadas por el Fondo Emprender en Colombia según rubro (2003-2018). (Datos de Cajigas et al., 2019)

De acuerdo con la entidad, de las 7.367 empresas apoyadas en todo el territorio nacional en el período 20032018, la mayor concentración se encuentra en rubros como agricultura, ganadería, caza y silvicultura (35\%), seguida de la industria manufacturera. Por tal motivo, los programas, líneas de acción y estrategias de fomento al emprendimiento deben orientarse a fomentar a aquellos sectores que se presentan menos favorecidos, como hoteles y restaurantes, comercio al por menor, y servicio comunitario, a fin de incrementar la participación en estas áreas productivas. Sin duda alguna, la educación empresarial, la cual se presenta como un vehículo para el desarrollo económico y social, genera un impacto de forma positiva en las acciones de emprendimiento, esto a través del impulso de no solo el espíritu emprendedor, sino que también de una cultura capaz de proveer nuevos modelos pedagógicos (Pitre et al., 2017; Hernández et al., 2017).

En consecuencia, es necesario crear mecanismos en donde los estudiantes mantengan contacto cercano con empresas, en un constante aprender haciendo que estimule el aprendizaje aplicado en contextos reales y que desde el inicio de sus carreras profesionales sean motivados a desarrollar proyectos empresariales propios como una opción viable (Ghina, 2014). En tal sentido, se evidencia un amplio espectro de desarrollo en busca de la generación de un marco educativo que sea coadyuvante a que el emprendimiento se establezca como eje transversal en la formación estudiantil (Von Graevenitz et al., 2010).

\section{Modelos formativos para los procesos de emprendimiento}

Timmons (1999) citado por Kuratko (2005), presenta su modelo homónimo, el cual está ampliamente desarrollado en la literatura científica como una guía factible en procesos formativos de emprendimiento. Fue desarrollado por el profesor Jeffrey Timmons quien ha sido reconocido por lograr la mayor cantidad de empresarios exitosos bajo su esquema. El modelo se fundamenta básicamente en los siguiente tres elementos: el mercado, (representado por las oportunidades), las personas, (representadas por el equipo emprendedor) y los recursos, (que consisten en los elementos de planificación y gestión eficiente de estos).

En este sentido, bajo el enfoque del modelo, se considera que las capacidades para emprender pueden ser adquiridas mediante procesos formativos, independientemente de las características del individuo, su formación previa o la etapa de vida que viva actualmente (Kuratko, 2005). En este orden de ideas, esquemáticamente, se expresan los elementos del modelo: oportunidad, personas y recursos, los cuales pueden apreciarse junto con su descripción como se observa en la figura 5 a continuación. 


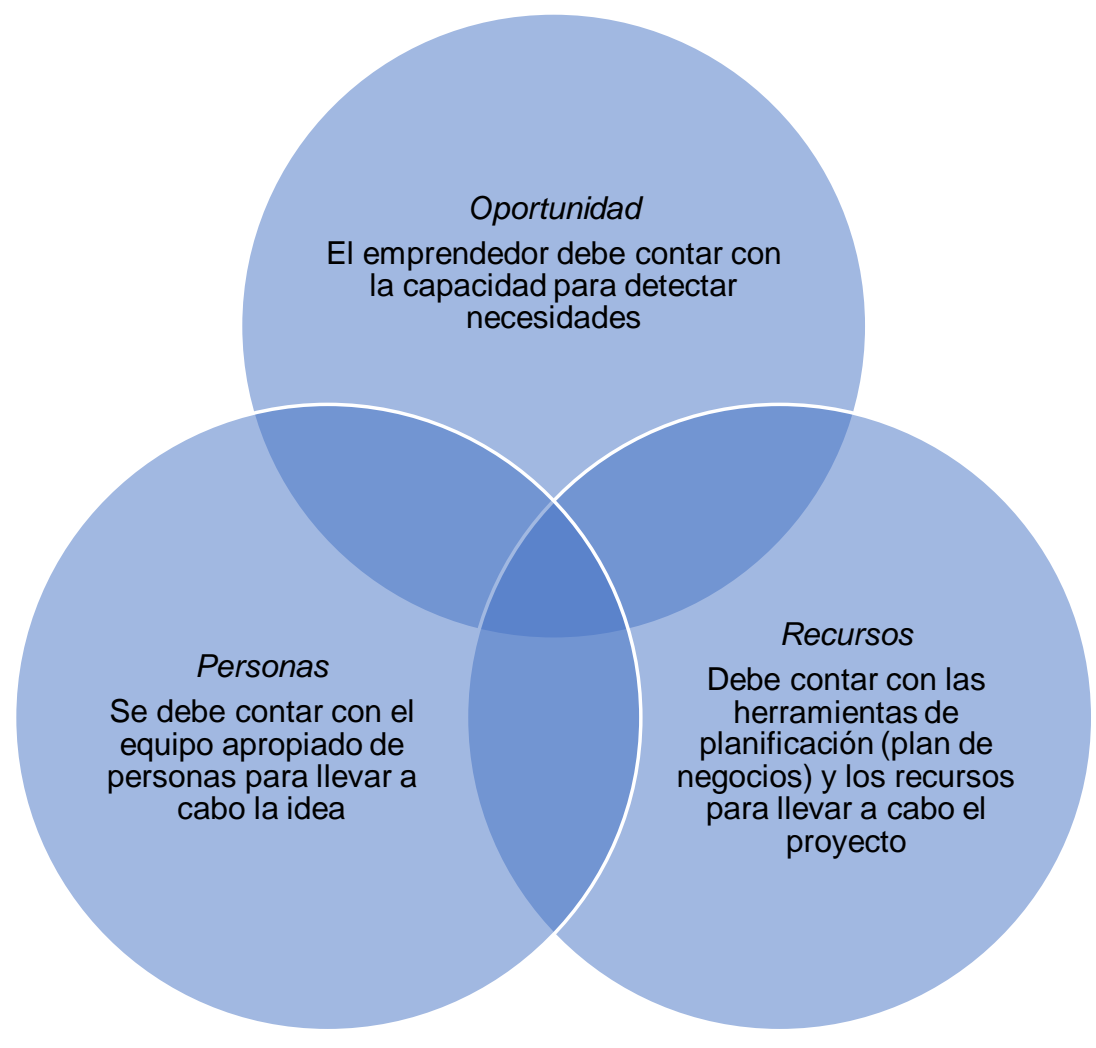

Fig 5. Modelo Timmons. (Adaptado de Kuratko, 2005)

Otro modelo que orienta al emprendedor en la consecución del proyecto productivo es el de Shapero (1984), citado por Zhang et al., (2014), el cual propone cinco elementos relacionados entre sí y fundamentado en dos competencias básicas, las relacionadas con los conocimientos que se requieren para poner en marcha una empresa y las competencias de orden individual, que no son más que todo el conjunto de habilidades, actitudes, valores, comportamientos que se requieren para ser un empresario de éxito. Las etapas propuestas por Shapero son las siguientes: motivacional (Gusto), la cual tiene que ver con los estímulos que el emprendedor recibe de su entorno y que le dan el impulso para enfrentar el proceso emprendedor, este entorno se compone por los sistemas educativos, la sociedad y la cultura. La segunda etapa es la situacional (oportunidad), donde el emprendedor, a partir de un análisis de su entorno, identifica una necesidad que puede ser cubierta (Zhang et al., 2014).

Seguidamente viene la etapa psicológica (decisión), en la cual se evalúa la prefactibilidad de la idea de negocios y se decide si continuar o no con el proceso dando paso a la fase analítica (Plan de negocios), en donde se desarrollan todos los elementos necesarios para poner en marcha la empresa, a través de un análisis profundo de aspectos como el mercado, finanzas, marketing, marco legal, entre otros. Por último, la fase económica (recursos), es aquella donde se genera el esfuerzo por obtener los recursos económicos para desarrollar el proyecto con base en la planificación establecida (Zhang et al., 2014).

De este modo, se tiene que los puntos contrastantes de estos dos modelos se destacan en la cantidad de elementos, pero se destaca que el modelo Timmons presenta dos de los aspectos que el Shapero, pero con el factor añadido de que Timmons prevé la necesidad del capital humano requerido, por lo que este modelo puede ser más fácilmente transmitido hacia los estudiantes que no tengan educación previa sobre emprendimiento, y se les establezcan esos 3 pilares como base en su proceso formativo, que son: personas, recursos y oportunidad, de manera que este modelo se puede implementar en procesos formativos introductorios y de prolongación mediana como: cursos de extensión, capacitaciones y diplomados (Kuratko, 2005). Por otro lado, Zhang et al., (2014) exponen el método Shapero el cual provee dos de los elementos que el modelo Timmons, pero además de contrastar en un tercer elemento, este se presenta más ampliado debido a que tiene cinco elementos en total, donde además de la oportunidad y los recursos, el modelo tiene un aspecto que expone la motivación inicial como punto de partida del proceso, otro donde abarca la evaluación de la idea de negocios previa al desarrollo de la planificación que sería el último elemento; este modelo según expresan Zhang et al., (2014) se presenta más digerible para aquellos estudiantes con bases educativas en materias de emprendimiento por lo que estipulan que se dentro de la educación superior debe dedicársele una importancia suficiente para su profundización al introducirlo dentro de la planificación académica. 


\section{CONCLUSIONES}

Dentro de las conclusiones más relevantes se tiene que no solo la universidad como institución es la que interviene y participa en el desarrollo de iniciativas empresariales. El entorno en el que se desarrollan los proyectos de emprendimiento está determinado también por políticas públicas vinculadas con las necesidades y demandas de la sociedad.

El entorno para el fomento del emprendimiento está conformado por diversos actores, entre estos, instituciones públicas y privadas, organizaciones no gubernamentales, instituciones educativas; cada uno representando los sectores más importantes de la sociedad: gobierno, empresas y comunidad. El emprendedor es quien identifica las oportunidades y se enfrenta a los riesgos del mercado, utilizando toda su experiencia y capacidad para desarrollar estrategias innovadoras para lograr, no solo la consolidación de su proyecto, sino una contribución importante al desarrollo y crecimiento económico y social.

La responsabilidad del éxito o fracaso de los proyectos de emprendimiento no deben recaer únicamente en el emprendedor como individuo, pues el contexto económico y social tan dinámico que se vive actualmente requiere de competencias y habilidades propias que le permitan al individuo enfrentar tales retos, pero no de forma unilateral, sino con apoyo de todos los actores involucrados.

Desde la academia es mucho el aporte que se puede hacer en términos de impulso y promoción del emprendimiento, con la ayuda de diversos actores del sistema económico y social es factible crear los mecanismos para la formación de los estudiantes universitarios en los procesos de emprendimiento. Este apoyo puede convertirse en un factor impulsor del desarrollo económico y social, pues diferentes estudios confirman que el emprendimiento y la educación están vinculados directamente al desarrollo de las naciones.

\section{AGRADECIMIENTOS}

A la puesta de marcha de un proyecto colaborativo entres las universidades públicas como lo son universidad de Sucre y Atlántico de la región caribe colombiana hacia una proyección hacia acreditación de programas académicos en el programa de pregrado Administración de empresas.

\section{REFERENCIAS}

Becker, M.C., Knudsen, T., y Swedberg, R., Schumpeter's Theory of Economic Development: 100 years of development, https://doi.org/10.1007/s00191-012-0297-x, Journal of Evolutionary Economics, 22, 917-933 (2012)

Bernal, P., Darío, I., y Argote-Cusi, M.L., Factores asociados a las empresas con potencial de crecimiento en la Universidad EAN, http://dx.doi.org/10.18359/rfce.1609, Revista Facultad de Ciencias Económicas: Investigación y Reflexión, 23(2), 97-108 (2015)

Bittencourt, M., Financial development and economic growth in Latin America: Is Schumpeter right?, https://doi.org/10.1016/j.jpolmod.2012.01.012, Journal of Policy Modeling, 34(3), 341-355 (2012)

Brown, C., y Thornton, M., How entrepreneurship theory created economics, Quarterly Journal of Austrian Economics, ISSN: 1098-3708, 16(4), 401-419 (2013)

Bucardo-Castro, M.A., Savedra-García, M.L., y Camarena-Adame, M.E., Hacia una comprensión de los conceptos de emprendedores y empresarios, https://doi.org/10.1016/j.sumneg.2015.08.009, Suma de Negocios, 6(13), 98-107 (2015)

Cajigas, M., Ramírez, E., y Ramírez, D., Capacidad de producción y sostenibilidad en empresas nuevas, Revista Espacios, ISSN: 0798 1015, 40(43), 15-29 (2019)

Campuzano-Vásquez, J., Espinoza-Freire, E., y Cedillo-Chalaco, L., La actividad emprendedora en la provincia de El Oro, una revisión desde la universidad, Universidad y Sociedad, ISSN: 2415-2897, 9(3), 73-81 (2017)

Carraher, S., Welsh, D., y Svilokos, A., Validation of a measure of social entrepreneurship, https://doi.org/10.1504/EJIM.2016.077421, European Journal of International Management, 10(4), 386-402 (2016)

Cantón-Castillo, L.C., García-Lira, A., y Luit-González, M.C., El Enfoque de Competencias para formar Emprendedores: Evaluación del modelo de la Universidad Autónoma de Yucatán, México, https://doi.org/10.18845/te.v8i2.1987, Tec Empresarial, 8(2), 29-40 (2014)

Denicolai, S., Hagen, B., y Pisoni, A., Be international or be innovative? Be both? The role of the entrepreneurial profile, https://doi.org/10.1007/s10843-015-0143-y, Journal of International Entrepreneurship, 13(4), 390-417 (2015)

Ghina, A., Effectiveness of entrepreneurship education in higher education institutions,

https://doi.org/10.1016/j.sbspro.2014.02.440, Procedia-Social and Behavioral Sciences, 115, 332-345 (2014)

Grisales-Ocampo, H.F., Propuesta de un modelo de apoyo al emprendimiento que genera sinergia con la innovación social, https://doi.org/10.22267/rtend.202101.131, Tendencias, 21(1), 157-174 (2020) 
Herrera-Guerra, C.E., y Montoya-Restrepo, L.A., El emprendedor: una aproximación a su definición y caracterización, https://doi.org/10.15765/pdv.v4i7.441, Punto de Vista, 4(7), 10-31 (2013)

Hernández, H.G., Cardona, D.A., y Del-Rio, J.L., Direccionamiento Estratégico: Proyección de la Innovación Tecnológica y Gestión Administrativa en las Pequeñas Empresas, http://dx.doi.org/10.4067/S0718-07642017000500003, Información Tecnológica, 28(5), 15-22 (2017)

Kuratko, D.F., The emergence of entrepreneurship education: Development, trends, and challenges, https://doi.org/10.1111/j.1540-6520.2005.00099.x, Entrepreneurship Theory and Practice, 29(5), $577-597$ (2005)

Niebles-Núñez, W.A., Hernández-Palma, H.G., y Cardona-Arbeláez, D., Gestión tecnológica del conocimiento: herramienta moderna para la gerencia de instituciones educativas, https://doi.org/10.19053/20278306.v7.n1.2016.5633, Revista de Investigación, Desarrollo e Innovación, 7(1), 25-36 (2016)

Numa, G., Jean-Baptiste Say on Free Trade, https://doi.org/10.1215/00182702-7803715, History of Political Economy, 51(5), 901-934 (2019)

Obschonka, M., Silbereisen, R.K., y Schmitt-Rodermund, E., Successful entrepreneurship as developmental outcome: A path model from a lifespan perspective of human development, https://doi.org/10.1027/1016-9040/a000075, European Psychologist, 16(3), 174 (2011)

Omerzel-Gomezelj, D., y Kušce, I., The influence of personal and environmental factors on entrepreneurs' performance, https://doi.org/10.1108/K-08-2012-0024, Kybernetes, 42 (6), 906-927 (2013)

Peñaherrera-León, M., y Cobos-Alvarado, F., La creatividad y el emprendimiento en tiempos de crisis, REICE, Revista Electrónica Iberoamericana sobre Calidad, Eficacia y Cambio en Educación, ISSN: 1696-4713, 10(2), $238-247$ (2012)

Pitre-Redondo, R., Cardona-Arbeláez, D., y Hernández-Palma, H., Proyección del emprendimiento indígena como mecanismo de competitividad en el postconflicto colombiano, https://doi.org/10.19053/20278306.v7.n2.2017.6068, Revista de Investigación, Desarrollo e Innovación, 7(2), 231-240 (2017)

Raposo, M., y do Paço, A., Entrepreneurship education: relationship between education and entrepreneurial activity, Psicothema, ISSN: 0214-9915, 23(3), 453-457 (2011)

Rodríguez-López, R., y Borges-Gómez, E., El perfil del emprendedor. Construcción cultural de la subjetividad laboral postfordista, http://dx.doi.org/10.5209/CRLA.60697, Cuadernos de Relaciones Laborales, 36(2), 265-284 (2018)

Salinas, R.F., y Osorio, L.R., Emprendimiento y Economía Social, oportunidades y efectos en una sociedad en transformación, CIRIEC-España, Revista de Economía Pública, Social y Cooperativa, ISSN: 0213-8093, (75), 128-151 (2012)

Singh-Sandhu, M., Fahmi-Sidique, S., y Riaz, S., Entrepreneurship barriers and entrepreneurial inclination among Malaysian postgraduate students, https://doi.org/10.1108/13552551111139656, International Journal of Entrepreneurial Behavior \& Research, 17(4), 428-449 (2011)

Suárez-Álvarez, J., y Pedrosa, I., Evaluación de la personalidad emprendedora: Situación actual y líneas de futuro, Papeles del Psicólogo, ISSN: 0214-7823, 37(1), 62-68 (2016)

Tunali, C.B., y Sener, S., The determinants of entrepreneurship in Turkey, https://doi.org/10.1016/j.procs.2019.09.099, Procedia Computer Science, 158, 648-652 (2019)

Von Graevenitz, G., Harhoff, D., y Weber, R., The effects of entrepreneurship education, https://doi.org/10.1016/j.jebo.2010.02.015, Journal of Economic Behavior \& Organization, 76(1), 90-112 (2010)

Wales, W.J., Entrepreneurial orientation: A review and synthesis of promising research directions, https://doi.org/10.1177/0266242615613840, International Small Business Journal, 34(1), 3-15 (2016)

Zhang, Y., Duysters, G., y Cloodt, M., The role of entrepreneurship education as a predictor of university students' entrepreneurial intention, https://doi.org/10.1007/s11365-012-0246-Z, International Entrepreneurship and Management Journal, 10(3), 623-641 (2014) 
